\title{
Chromosomal Inversion Process
}

National Cancer Institute

\section{Source}

National Cancer Institute. Chromosomal Inversion Process. NCI Thesaurus. Code C89778.

Any type of genetic recombination involving rearrangement of segments of DNA within a single chromosome, such that a segment of the chromosome is reversed from the original orientation. Chromosomal inversion often occurs as the result of nonhomologous end-joining of broken DNA strands, and is involved in maintaining cell viability at the expense of long term genomic stability. This process may be involved in infertility. 